\title{
Epidemiologia da Doença de Ménière: Revisão da Literatura
}

\section{Epidemiology of Ménière's Disease: Literature Review}

Diogo Ribeiro ${ }^{1}$, Cristina Caroça $a^{1,2}$, João Paço ${ }^{1,2}$

Autor Correspondente: Diogo Ribeiro [diogo.ribeiro@jmellosaude.pt] Avenida Infante Santo, n³4, $5^{\circ}$ Piso, 1350-179 Lisboa, Portugal

\section{RESUMO}

A doença de Ménière é uma patologia crónica do ouvido interno caracterizada por sintomas vestibulares episódicos associados a hipoacusia neurossensorial, acufeno e plenitude auricular. Tendo sido descrita há mais de 150 anos por Prosper Ménière, ainda hoje em dia a sua epidemiologia continua a ser um enorme desafio para os profissionais que tentam compreender melhor esta doença.

Desde a Segunda Guerra Mundial que várias sociedades e grupos de estudo têm tentado aprofundar o conhecimento desta patologia, nomeadamente a sua prevalência e incidência. Contudo, devido à dificuldade de uniformização dos critérios de diagnóstico, estes valores apresentam grande variabilidade.

Com o seu aparecimento na meia-idade, a doença de Ménière apresenta-se inicialmente como uma doença unilateral, não se encontrando tendência para uma maior expressividade quanto à lateralidade. Com o passar dos anos, verifica-se uma tendência clara para a bilateralidade da doença de Ménière, em que o tempo de envolvimento do ouvido contralateral pode variar de dois meses a mais de 20 anos. Por outro lado, com o passar dos anos, ocorre uma redução no impacto dos sintomas, nomeadamente através de mudanças no estilo de vida e tratamento médico ou cirúrgico. O sistema imunológico, a enxaqueca, a alergia e o sistema nervoso autónomo estão também associados aos doentes com doença de Ménière.

De um modo geral, é consensual que existe um maior predomínio da doença de Ménière na raça caucasiana, quanto ao género, um ligeiro predomínio da doença de Ménière no sexo feminino é reportado na grande maioria dos estudos. Evidências epidemiológicas e genómicas suportam uma suscetibilidade genética.

PALAVRAS-CHAVE: Comorbilidade; Doença de Ménière/diagnóstico; Doença de Ménière/epidemiologia; Doença de Ménière/genética 


\begin{abstract}
Ménière's disease is a chronic pathology of the inner ear characterized by episodic vestibular symptoms associated with sensorineural hypoacusis, tinnitus and aural fullness. Having been described more than 150 years ago, by Prosper Ménière nowadays its epidemiology continues to be a great challenge for professionals trying better understand this disease.

Since World War II several societies and study groups have tried to deepen the knowledge of this pathology, namely its prevalence and incidence. However, due to the difficulty of standardization of diagnostic criteria, these values present great variability.

With its onset in middle age, Ménière's disease initially presents itself as a unilateral disease without tendency for greater expressiveness regarding laterality. Over the years, there is a clear trend toward bilateral Ménière's disease, in which the time of contralateral ear involvement may vary from two months to more than twenty years. On the other hand, over the years, there was a reduction on the impact of the symptoms, namely through changes in lifestyle and medical or surgical treatment. The immune system, migraine, allergy and autonomic nervous system are also associated with Ménière's disease.

In general, it is agreed that there is a greater predominance of Ménière's disease in the Caucasian race, as for gender, a slight predominance of female is reported in the vast majority of studies. Epidemiological and genomic evidence supports a genetic susceptibility.
\end{abstract}

KEYWORDS: Comorbidity; Ménière's Disease/diagnosis; Ménière's Disease/epidemiology; Ménière's Disease/genetics

\section{INTRODUÇÃO}

Apesar da doença de Ménière (DM) ter sido descrita há mais de 150 anos por Prosper Ménière, ainda hoje em dia a sua epidemiologia continua a ser um enorme desafio para os profissionais que tentam compreender meIhor esta doença. ${ }^{1}$

Este desafio deve-se em grande parte à dificuldade no diagnóstico, por um lado, por ainda não ser uma doença totalmente conhecida e, por outro lado, a dificuldade do seu diagnóstico numa fase preliminar, onde não há a presença da tríade sintomática típica. ${ }^{2}$ Ao desafio junta-se ainda a falta de critérios (validados ou bem definidos), bem como as diferentes metodologias utilizadas nos diversos estudos epidemiológicos (transversais ou retrospetivos), o que leva a uma grande variabilidade de resultados obtidos. ${ }^{3-7}$

Com vista a encontrar uma solução que permitisse que diferentes instituições pudessem comparar os seus resultados mediante um sistema uniforme e objetivo, o "Comité de Audição e Equilíbrio" da Academia Americana de Oftalmologia e Otorrinolaringologia publica, em 1972, o primeiro consenso sobre o diagnóstico da DM que, posteriormente, é modificado pela American Academy of Otolaryngology-Head and Neck Surgery (AAO-HNS) em 1985 e 1995.8-10

No Japão é criado um "Comité de Investigação" da DM que viria a ser reorganizado mais tarde, em 1980, para o "Comité de Investigação de Doenças Vestibulares Periféricas" pela Sociedade Japonesa de Pesquisa para o Equilíbrio (JSER) e, à semelhança da AAO-HNS, estipula recomendações para o diagnóstico da DM. ${ }^{11}$
Mais recentemente, a Sociedade Barany em colaboração com as JSER, AAO-HNS, Academia Europeia de Otologia e Otoneurologia e Sociedade Coreana para o Equilíbrio estabelecem novos critérios de diagnóstico para a DM. ${ }^{12}$

\section{PREVALÊNCIA E INCIDÊNCIA}

A prevalência e incidência da DM têm sido estudadas desde a Segunda Guerra Mundial. Contudo, a sua interpretação torna-se equívoca quando se pretende comparar os diversos estudos publicados. Para além dos fatores já referidos anteriormente, que levam à dificuldade em se determinar com precisão os dados epidemiológicos, também os conceitos de prevalência e incidência foram abordados das mais variadas formas ao longo dos anos..$^{13}$

Um dos primeiros estudos publicados sobre epidemiologia da DM foi conduzido em Inglaterra por Cawthorne e Hewlett, onde apresentam uma série de 900 casos identificados com DM. No entanto, tendo em consideração que com base na sua amostra não chegariam a um valor real da incidência da DM, baseiam-se nos registos médicos de oito clínicas, entre março de 1953 e março de 1954, abrangendo uma população de 27365 habitantes e encontram 43 casos de DM, obtendo assim uma incidência de 15714 casos por 100000 habitantes. ${ }^{14}$

Mais tarde, Stahle et al analisam os registos clínicos em duas cidades suecas e identificam 257 casos de DM determinando, para o ano de 1973, uma incidência de 46 casos por 100000 habitantes. $^{6}$

Wladislavosky-Waserman et al, numa análise aos estudos anteriores referem, que a incidência está aumenta- 
da, muito provavelmente devido ao valor a que chegaram ser uma combinação da prevalência e incidência. Referem ainda que os valores apurados no estudo sueco não incluem dados das clínicas privadas, o que na altura correspondia a aproximadamente $23 \%$ da população sueca. ${ }^{3}$

Da Finlândia surgem dois estudos que apresentam prevalências tão díspares como 43,2 casos por 100000 habitantes num e 513 casos por 100000 habitantes no outro. ${ }^{13,15}$ Estes resultados poderiam ser explicados com base nas diferenças geográficas e étnicas que podem existir dentro de um mesmo país mas, quando se analisam com detalhe ambos os estudos, percebemos que estas diferenças se devem essencialmente às diferentes metodologias aplicadas em cada um dos estudos. ${ }^{16}$

Ambos os estudos são analisados por Radtke et al. No primeiro estudo, acreditam que a verdadeira prevalência é presumivelmente mais alta, tendo em conta que muitos casos de DM não estão refletidos no estudo. Já no segundo estudo, Havia et al apontam para uma sobrestimação dos valores apurados uma vez que o questionário utilizado apresenta várias falhas. ${ }^{17}$ Por sua vez, Radtke et al realizam um estudo na Alemanha onde determinam uma prevalência de 120 casos por 100000 habitantes, numa amostra aleatória de 4869 habitantes, aplicando os critérios estabelecidos pela AAO-HNS (1995).17

Num dos estudos mais recentes realizados no RU por Tyrrel et al, tendo como base o Biobanco no RU, é determinada uma prevalência de 270 casos por 100000 habitantes. $\bigcirc$ estudo, considerado um dos maiores sobre a epidemiologia da DM, apresenta um grupo de controlo de aproximadamente meio milhão, com 1376 casos de DM identificados entre 2006 e $2010 .{ }^{18}$

Uma das fontes mais importantes sobre dados epidemiológicos da DM provém de estudos realizados no Japão. Naito reporta que a frequência da DM no Japão aumentou drasticamente desde a Segunda Guerra Mundial, passando de 100 casos por 100000 habitantes, em 1941, para 3400 casos por 100000 habitantes em 1960, concluindo que este aumento se deveu à altera- ção do modo de vida dos japoneses devido à ocidentalização provocada no período de guerra. ${ }^{19}$

Mais tarde, Nakae et al, publicam um estudo com duas abordagens diferentes. Os valores observados apresentam grande variabilidade, obtendo-se com a primeira abordagem uma prevalência de 17 casos por 100000 habitantes e na segunda 3,5 casos por 100000 habitantes. ${ }^{20}$ Este último valor encontrado é demasiado baixo para o expectável, levando a crer que esta subestimação da prevalência se deve à exclusão dos doentes de clínicas ambulatórias e ao maior rigor nos critérios utilizados para o diagnóstico da DM nos hospitais universitários. ${ }^{5}$

Watanabe et al, publicam os estudos regionais realizados pela Associação Médica da Prefeitura de Toyama e a prevalência mantém-se constante em 16-17 casos por 100000 habitantes, num período compreendido entre 1974 e 1990. Contudo, os autores especulam que a real prevalência da DM pode estar sobrestimada devido aos critérios de diagnóstico usados pelos estudos conduzidos pela Associação Médica da Prefeitura de Toyama. ${ }^{11}$

Por fim, Shojaku et al, realizam um estudo retrospetivo entre o período de 1990 e 2004 no distrito de Nishikubiki e, entre 1980 e 2004, na Universidade Médica e Farmacêutica de Toyama identificando 375 doentes com DM definida (JSER), 50 em Nishikubiki e 325 em Toyama. A média anual da prevalência e incidência em Toyama foi de 34,5 e cinco casos respetivamente por 100000 habitantes. Em Nishikubiki a prevalência foi de 38 casos por 100000 habitantes. ${ }^{21}$

Um dos estudos mais citados sobre a epidemiologia da DM foi realizado nos EUA em 1984 por Wladislavosky-Waserman et al. Baseando-se nos registos médicos da Mayo Clinic e do grupo médico Olmsted, os principais fornecedores de assistência médica para os 40000 habitantes da cidade de Rochester, chegam a uma prevalência, no dia 1 de janeiro de 1980, de 119 casos de DM, ou seja, o correspondente a 218,2 casos por 100000 habitantes. A taxa de incidência total reportada para o ano de 1980 foi de 15,3 casos por 100000 habitantes. ${ }^{3}$

TABELA 1. Prevalência e incidência da doença de Ménière na Europa.

\begin{tabular}{|l|l|l|l|l|l|} 
Autores & País & No de Casos & $\begin{array}{l}\text { Critérios de } \\
\text { Diagnóstico }\end{array}$ & $\begin{array}{l}\text { Prevalência } \\
\text { por } 100000 \\
\text { Habitantes }\end{array}$ & $\begin{array}{l}\text { Incidência } \\
\text { por } 100 \text { 000 } \\
\text { Habitantes }\end{array}$ \\
\hline Cawthorne e Hewlett (1954) ${ }^{14}$ & Inglaterra & 43 & N/D & N/D & 157,14 \\
\hline Stahle et al $(1978)^{6}$ & Suécia & 257 & N/D & N/D & 46 \\
\hline Kotimaki et al (1999) 13 & Finlândia & 131 & AAO-HNS (1995) & 43,2 & 4,3 \\
\hline Havia et al $(2005)^{15}$ & Finlândia & 16 & AAO-HNS (1995) & 513 & N/D \\
\hline Radtke et al (2008)17 & Alemanha & 4 & AAO-HNS (1995) & 120 & N/D \\
\hline Tyrrel et al $(2014)^{18}$ & Inglaterra & 1.376 & N/D & 270 & N/D \\
\hline
\end{tabular}


TABELA 2. Prevalência e incidência da doença de Ménière no Japão.

\begin{tabular}{|l|l|l|l|l|}
\hline Autores & No de Casos & $\begin{array}{l}\text { Critérios de } \\
\text { Diagnóstico }\end{array}$ & $\begin{array}{l}\text { Prevalência } \\
\text { por } 100 \text { 000 } \\
\text { Habitantes }\end{array}$ & $\begin{array}{l}\text { Incidência } \\
\text { por } 100 \text { 000 } \\
\text { Habitantes }\end{array}$ \\
\hline Nakae et al $(1980)^{20}$ & N/D & N/D & 73 & N/D \\
\hline Nakae et al $(1980)^{20}$ & N/D & N/D & 3,5 & N/D \\
\hline Watanabe et al $(1995)^{11}$ & 955 & JSER & $16 / 17$ & N/D \\
\hline Shojaku et al $(2005)^{21}$ & 50 & JSER & 38 & N/D \\
\hline Shojaku et al $(2005)^{21}$ & 325 & JSER & 34,5 & 5 \\
\hline
\end{tabular}

TABELA 3. Prevalência e incidência da doença de Ménière nos Estados Unidos da América.

\begin{tabular}{|l|l|l|l|l|}
\hline Autores & $N^{\circ}$ de Casos & $\begin{array}{l}\text { Critérios de } \\
\text { Diagnóstico }\end{array}$ & $\begin{array}{l}\text { Prevalência por } 100 \\
000 \text { Habitantes }\end{array}$ & $\begin{array}{l}\text { Incidência por } 100 \text { 000 } \\
\text { Habitantes }\end{array}$ \\
\hline Wladislavosky-Waserman et al $(1984)^{3}$ & 180 & AAO-HNS (1972) & 218,2 & 15,3 \\
\hline Harris e Alexander $(2010)^{5}$ & 473000 & N/D & 190 & N/D \\
\hline
\end{tabular}

TABELA 4. Prevalência e incidência da doença de Ménière em África.

\begin{tabular}{|l|l|l|l|l|}
\hline Autores & No de Casos & $\begin{array}{l}\text { Critérios de } \\
\text { Diagnóstico }\end{array}$ & $\begin{array}{l}\text { Prevalência } \\
\text { por } 100000 \text { Habitantes }\end{array}$ & $\begin{array}{l}\text { Incidência } \\
\text { por } 100000 \text { Habitantes }\end{array}$ \\
\hline Okafor $(1984)^{23}$ & 20 & N/D & 400 & N/D \\
\hline Brobby (1992) ${ }^{24}$ & 59 & N/D & N/D & 320 \\
\hline Ibekwe e ljaduola $(2007)^{25}$ & 25 & N/D & 218,09 & N/D \\
\hline
\end{tabular}

Celestino e Ralli, em 1991, apontam que um terço dos casos incluídos no estudo americano apresentam vertigem recorrente sem sintomas cocleares, o que não vai de encontro aos critérios estabelecidos pela AAO-HNS (1985), estando assim sobrestimada a prevalência. ${ }^{22}$ Mais tarde, Harris e Alexander determinam que apenas 41\% dos doentes identificados com DM cumprem os critérios da AAO-HNS (1995), chegando a conclusão semelhante sobre uma possível sobrestimação dos valores apresentados. $^{5}$

Harris e Alexander, num estudo mais recente, efetuado entre 2005 e 2007, numa base de dados de mais de 60 milhões de doentes que incorpora 97 companhias de seguro que cobrem praticamente $25 \%$ da população dos EUA, encontraram 473000 casos de DM obtendo uma prevalência estimada de 190 casos por 100000 habitantes. ${ }^{5}$

Do continente Africano destacam-se três estudos realizados sobre a epidemiologia da DM. O primeiro, realizado por Okafor em 1984, determina uma prevalência da DM de 400 casos por 100000 habitantes na África Ocidental. ${ }^{23} \mathrm{O}$ segundo, realizado por Brobby oito anos mais tarde, documenta uma incidência de 320 casos por 100 000 habitantes. ${ }^{24}$ Por fim, o estudo mais recente realizado no continente Africano foi efetuado por Ibekwe e Ijaduola em 2007, onde realizaram um estudo retrospetivo durante 10 anos, com um total de 11463 doentes vistos no departamento de ORL do University College
Hospital, diagnosticando 25 casos de DM. A prevalência encontrada foi de 218,09 casos por 100000 habitantes, valor ligeiramente abaixo dos achados nos estudos prévios. $^{25}$

\section{DOENÇA DE MÉNIÈRE NA INFÂNCIA}

Existem relatos esporádicos de casos de DM em idades inferiores a 20 anos. $^{6}$ Alguns desses casos reportados com doença de Ménière infantil (DMI) surgem associados a fatores desencadeantes da hidropsia endolinfática, nomeadamente a perda de audição hereditária, embriopatias, infeções severas do ouvido/cérebro ou traumas do ouvido/cabeça, ao invés da típica DM idiopática. ${ }^{26-28}$

Como no adulto, também na criança o diagnóstico da DM é de extrema dificuldade, por um lado, por algumas patologias levarem a um diagnóstico errado e, por outro, porque as crianças, na maioria das vezes, não conseguem referir os sintomas. Contudo, mesmo estando em falta esta informação, é razoável considerar crianças com crises de vertigem idiopática recorrentes, com mais de 20 minutos e com audiogramas a documentar perda de audição neurossensorial unilateral com flutuação nas frequências graves, como tendo DM. ${ }^{29}$

Nos EUA estima-se que 3\% dos casos de DM se encontram em idade pediátrica. ${ }^{27}$ Harris determina uma prevalência de nove casos por 100000 habitantes. ${ }^{5}$ Por sua 
vez, Hausler determina que 1\% dos casos de DM são crianças e 1,5\% das crianças com vertigem apresentam DM típica. ${ }^{26}$

Akagi et al determinam uma incidência da DMI, no seu departamento, de 2,9\%. ${ }^{30} \mathrm{~A}$ incidência da DM em crianças é 100 vezes menor quando comparada com a população adulta. ${ }^{26}$

\section{DOENÇA DE MÉNIÈRE NO IDOSO}

A prevalência e incidência da DM no idoso não é tão incomum como se pensava inicialmente, podendo aparecer como uma nova doença ou como doença de longa data reativada. ${ }^{31}$

Harris e Alexander reportam uma prevalência de 440 casos para 100000 habitantes para o grupo acima dos 65 anos, valor acima dos reportados em idades inferiores no seu estudo. 5 Também Havia et al reportam um aumento da prevalência acima dos 60 anos, sendo o pico da prevalência na faixa etária dos 61 aos 70 anos, 1709 casos por 100000 habitantes, com uma ligeira redução no grupo com idade superior a 70 anos, 1064 casos por 100000 habitantes. ${ }^{15}$

Shojaku et al constatam que a incidência da DM, em idades superiores a 65 anos, teve um aumento significativo durante o período de 1980 a 2004 e atribuem este aumento não só a uma melhoria na qualidade de vida do idoso, mas também a um aumento da população nessa faixa etária que continua ativa. ${ }^{21}$

\section{IDADE DE APARECIMENTO DOS SINTOMAS E IDADE DE DIAGNÓSTICO}

A grande maioria dos estudos aponta para o aparecimento da DM na meia-idade, ou seja, na quarta e quinta década de vida. ${ }^{3,11,14,32}$

Sendo que o aparecimento da DM, na maioria dos casos, se manifesta apenas com um sintoma, existe um intervalo de tempo até o diagnóstico definitivo ser realizado. Esse intervalo de tempo até ao aparecimento da tríade sintomática típica é variável, uma vez que os sintomas podem estar em remissão por longos períodos de tempo. 3,32

Tanto no estudo de Wladislavosky-Waserman et al como no de Friberg et al, a média entre a idade de aparecimento dos sintomas e a idade de diagnóstico é de, aproximadamente, três anos. ${ }^{3,32}$

\section{BILATERALIDADE NA DOENÇA DE MÉNIÈRE}

Existe uma tendência clara para a DM se tornar bilateral. Se nos estudos iniciais esta situação não se verificava, isso devia-se à janela de análise dos estudos publicados se basearem num curto espaço de tempo. Com o aparecimento mais recente de estudos em que existe um aumento dos anos de follow-up torna-se evidente um aumento de casos de doença de Ménière bilateral (DMB). ${ }^{33}$ Para além do tempo de análise já referido, uma vez mais os critérios de diagnóstico utilizados, bem como o local onde o estudo tem lugar, contribuem para uma grande variabilidade de resultados obtidos que se situam entre 5,3 e $47 \% .{ }^{16,32-34}$

House et al determinam uma prevalência de casos de DMB de 24\%, sendo o aparecimento simultâneo em ambos os ouvidos em 11\% dos casos e, em 14\% dos casos, um aparecimento progressivo. ${ }^{35}$ Num estudo a decorrer no nosso serviço, analisado pela última vez no final de 2015, numa amostra de 151 casos identificados com DM, segundo os critérios da AAO-HNS (1995), encontramos uma percentagem de casos de DMB de 12,6\%.

\section{TEMPO DE ENVOLVIMENTO DO OUVIDO CONTRALATERAL}

O tempo de envolvimento do ouvido contralateral pode variar de dois meses a mais de 20 anos. ${ }^{35}$ Paparella e Griebie reportam que 50\% dos casos de DMB têm início de envolvimento do ouvido contralateral durante os primeiros dois anos após o início do aparecimento dos sintomas. Dos restantes, $27 \%$ têm início nos primeiros cinco anos e os remanescentes 23\% até um intervalo máximo de 25 anos. ${ }^{36}$ Também Enander e Stahle deter-

TABELA 5. Idade de aparecimento e idade de diagnóstico.

\begin{tabular}{|l|l|l|}
\hline Autores & $\begin{array}{l}\text { Média de Idade de Aparecimento } \\
\text { (Intervalo) }\end{array}$ & $\begin{array}{l}\text { Média de Idade de Diagnóstico } \\
\text { (Intervalo) }\end{array}$ \\
\hline Cawthorne, Hewlett (1954) ${ }^{14}$ & 44 & N/D \\
\hline Wladislavosky-Waserman et al (1984) ${ }^{3}$ & $50(11-88)$ & $53(15-88)$ \\
\hline Friberg et al (1984) ${ }^{32}$ & $38,2(8-68)$ & $41,6(8-68)$ \\
\hline Watanabe et al (1995) & 42,2 (Homens) & N/D \\
\hline
\end{tabular}


minam que 36,2\% dos casos ocorrem depois de um intervalo superior a cinco anos. ${ }^{37}$

A contrastar estes achados temos um estudo realizado por Thomas e Harrison que afirmam que a grande maioria dos casos de doença de Ménière unilateral (DMU) que passam para DMB manifestam-se invariavelmente nos primeiros cinco anos. Dos 30 casos em que foi efetuado um seguimento de pelo menos 10 anos, 70\% viram aparecer o início dos sintomas no ouvido contralateral nos primeiros dois anos. ${ }^{38}$

House et al, determinam uma média para o intervalo de tempo do envolvimento do ouvido contralateral de 7,6 anos, variando de dois meses a 27 anos, sendo que 48\% dos casos ocorrem nos primeiros cinco anos desde o aparecimento da DMU. ${ }^{35}$ Também Stahle e Bergman determinam uma média de idade de envolvimento do ouvido contralateral semelhante, 8,7 anos. $^{39}$

\section{OUVIDO DIREITO VS OUVIDO ESQUERDO}

Nenhum dos lados parece apresentar uma maior predisposição para a DM. No estudo de Wladislavosky-Waserman et al, no total de 180 casos de DM, 34,4\% eram bilaterais, 31,6\% no ouvido direito e os restantes 33,8\% no ouvido esquerdo, não encontrando diferenças estatisticamente significativas. ${ }^{3}$ Stahle num estudo de coorte de 356 casos de DM que foram submetidos a cirurgia, $46 \%$ dos casos correspondiam ao ouvido direito, 44,4\% ao ouvido esquerdo e os restantes 9,6\% eram casos bilaterais, não se verificando também uma propensão para nenhum dos lados. ${ }^{40} \mathrm{Na}$ amostra por nós recolhida encontrámos 37,1\% de casos de DM no ouvido direito, $50,3 \%$ no ouvido esquerdo e $12,6 \%$ de casos bilaterais.

\section{ETNIA}

É consensual, de um modo geral, entre os vários investigadores que abordam as diferenças étnicas, que existe um maior predomínio da DM na raça caucasiana. ${ }^{41} \mathrm{Ca}$ parosa realiza um estudo onde comprova essa predominância da raça caucasiana, mostrando a ocorrência apenas ocasional na raça negra. ${ }^{42}$ Kitahara et al, demonstram que a incidência da DM em caucasianos americanos quando comparada com a raça negra americana é de dois para um. ${ }^{43}$ Tyrrel et al, determinam um rácio de 1,7:1 para a raça caucasiana quando comparada com as restantes raças. ${ }^{18}$

A contrastar estes achados, estudos provenientes do continente Africano defendem que a DM está subdiagnosticada entre a raça negra e apresentam como causas para essa diferença observada a localização geográfica, que leva a uma limitação ao acesso às instalações médicas, as próprias limitações das instalações médicas, as condições sanitárias e por fim a falta de meios de diagnóstico diferencial. De acordo com estes estudos, a prevalência da DM na raça negra situa-se muito próxima da encontrada para a raça caucasiana. ${ }^{23-25}$

Tanto a raça asiática como a hispânica apresentam uma menor incidência da DM quando comparadas com a raça caucasiana. ${ }^{21,41,44}$

\section{GÉNERO}

Um ligeiro predomínio da DM no sexo feminino é reportado na grande maioria dos estudos. ${ }^{3,5,6,15,18}$ Por outro lado, existem estudos onde não ocorrem diferenças significativas quanto ao género e noutros até um ligeiro predomínio do sexo masculino. ${ }^{14,22,40}$

Segundo Watanabe, a relação feminino-masculino mudou de uma predominância no sexo masculino para uma predominância no sexo feminino durante o período compreendido entre 1975 e 1990. ${ }^{11}$ Wladislavosky-Waserman et al, reportam um rácio geral feminino/masculino de 1,57:1, não sendo este estatisticamente significativo, contudo observam uma diminuição progressiva no sexo feminino durante o período de 1951 até 1980. ${ }^{3}$ Havia et al, encontram um rácio feminino/masculino de 4,3:1, no entanto, sentem que existe uma maior relutância no sexo masculino em participar neste tipo de estudos podendo contribuir, em certa parte, para um predomínio no sexo feminino. ${ }^{15}$ Já Tyrrel et al, encontram um rácio feminino/ masculino de 1,4:1. ${ }^{18}$ Valor semelhante foi também o encontrado na nossa amostra, 1,44:1, sendo 58,9\% dos casos do sexo feminino e 41,1\% masculino.

\section{FATORES GENÉTICOS E HISTÓRIA FAMILIAR}

Tendo em conta os achados epidemiológicos da DM, parece patente a existência de uma predisposição genética. Para começar, vários estudos apontam que 3 a 20\% dos casos de DM é familiar. ${ }^{45,46}$ Em adição aos casos familiares, as diferenças na distribuição geográfica e diferentes incidências da DM, no género e raça, sugerem que os fatores genéticos são significativos. ${ }^{13,23,41}$ Uma frequência mais elevada de doenças autoimunes sistémicas observada neste grupo reforça, também ela, esta presunção. ${ }^{47}$ Mizukoshi et al, num estudo realizado no Japão, encontram 5,8\% de casos numa coorte de 520 doentes de DM, que apresentam um familiar próximo com a mesma patologia. ${ }^{48}$ Klockars e Kentala encontram uma prevalência 


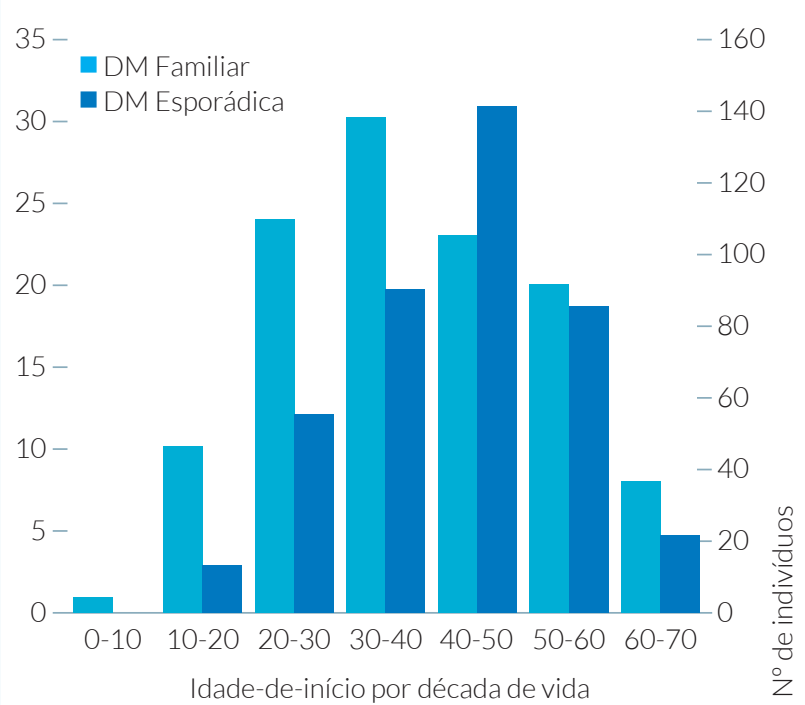

FIGURA 1. Comparação da idade de aparecimento da DM esporádica em comparação com a DMF.

Adaptado de Morrison AW, et al. Familial Ménière's disease: clinical and genetic aspects. J Laryngol Otol. 2009;123:29-37.44

de 15\% de casos de doença de Ménière familiar (DMF) na Finlândia, sendo a grande maioria do sexo feminino. ${ }^{49}$

Morrison reporta pela primeira vez antecipação na DMF, ou seja, um aparecimento mais precoce na DMF tendo em conta a idade, assim como uma tendência no aumento da severidade e intensidade dos ataques nas gerações sucessivas de DMF. ${ }^{50}$ Estes achados foram suportados mais tarde por estudos efetuados por Fung et al, Oliveira et al e Klockars e Kentala. ${ }^{49,51,52}$ Contudo, em algumas famílias esta antecipação não foi observada. ${ }^{51}$ Morrison et al documentam uma antecipação, da quinta para a quarta década de vida, no aparecimento da DMF quando comparada com a DM esporádica. ${ }^{44}$

Apesar da grande maioria das famílias descritas nos diversos estudos apresentar uma herança autossómica dominante, com penetrância incompleta de aproximadamente 60\%, a DMF apresenta heterogeneidade genética, com padrões de herança recessivos e mitocondriais descritos em algumas famílias. ${ }^{49,53}$

\section{FATORES INDIVIDUAIS}

Tyrrel et al encontram uma associação entre a DM e o peso, determinando um rácio de 1,2:1 em pessoas com excesso de peso, 1,3:1 em pessoas obesas e 1,7:1 para pessoas com obesidade mórbida. ${ }^{18}$

Ibekwe e ljadoula estratificam em classes sociais os casos com DM e determinam que 68\% dos casos pertencem a uma classe social baixa. ${ }^{25}$ Tyrrel et al, afirmam no seu estudo que, devido à dimensão da amostra, esta fornece uma robusta evidência que a DM, no momento do re- crutamento, tem uma maior prevalência em pessoas que apresentam um baixo nível socioeconómico. Antecipam também que pode existir um impacto no nível socioeconómico depois do diagnóstico da DM, pela possível redução na capacidade do indivíduo conseguir ganhar ou trabalhar a tempo integral. Para finalizar, observam uma associação entre o nível de escolaridade e a DM, que é potencialmente sugestiva de que exposições e experiências negativas relacionadas com um baixo nível socioeconómico contribuem para a patologia da DM. ${ }^{18}$

Numa tentativa de clarificar o perfil psicológico dos doentes com DM, Coker et al, demonstram que 80\% dos casos com sintomas vestibulares "ativos" apresentam depressão em comparação com 32\% no grupo em que os sintomas estavam "inativos". ${ }^{4}$ Friberg e Stahle apresentam duas potenciais alternativas da influência psicológica na DM. Se os distúrbios psicológicos são secundários às alterações vestibulares ocorre um efeito somatopsíquico, ao invés, se os distúrbios psicológicos antecedem as alterações vestibulares esse efeito é psicossomático. ${ }^{55}$ Já Tyrrel et al fornecem evidências que os doentes com DM, numa fase inicial, têm maior probabilidade de apresentarem uma má saúde mental, verificando-se uma redução no impacto dos sintomas em 85\% dos casos de DM à medida que os anos passam, nomeadamente através de mudanças no estilo de vida e tratamento médico ou cirúrgico. ${ }^{18}$

\section{DISTRIBUIÇÃO OCUPACIONAL}

Não existem muitos estudos publicados que relacionam a DM e as diferentes ocupações. Num estudo realizado por Cellestino e Ralli foi encontrada uma incidência superior em 4,3 vezes entre os funcionários do hospital quando comparada com a remanescente população do estudo. Contudo, este aumento não pode ser atribuído às diferenças entre os diversos grupos profissionais incluídos no estudo mas sim ao facto de existir uma maior acessibilidade às instalações médicas, bem como à facilidade que este grupo profissional tem em obter examinação médica. ${ }^{22}$

Uma incidência mais alta da DM entre ocupações profissionais e de gestão foi reportada por Watanabe et al. ${ }^{11}$ Um estudo anterior realizado no Japão reporta uma incidência aparentemente superior entre técnicos ou trabaIhadores de "colarinho branco" quando comparada com agricultores, pescadores, operários e aqueles envolvidos nos chamados trabalhadores de "colarinho azul". ${ }^{56}$

Por outro lado, Wladislavosky-Waserman et al não encontram nenhuma preponderância estatisticamente significativa para as diversas ocupações encontradas no seu estudo. ${ }^{3}$ 


\section{COMORBILIDADES}

O sistema imunológico (SI), a enxaqueca e a alergia, foram implicadas previamente numa série de estudos com a DM. ${ }^{25,47,57}$ Tyrrel et al, corroboram estes achados prévios e acrescentam, pela primeira vez, uma associação entre a DM e o sistema nervoso autónomo (SNA). ${ }^{18}$ Também uma elevada prevalência para doenças autoimunes sistémicas foi encontrada. ${ }^{47}$ Para além da associação a doenças autoimunes sistémicas, 60\% dos doentes com DM apresentam anticorpos séricos contra as proteínas do ouvido interno, bem como tiroidite autoimune. ${ }^{58}$

A associação entre a alergia e a DM foi pela primeira vez identificada na literatura num estudo efetuado em 1923 por Duke. ${ }^{59}$ Alguns autores defendem que 30\% dos doentes com DM apresentam alergia alimentar. ${ }^{60} \mathrm{~A}$ grande maioria da literatura corrente, que suporta esta associação, provém do trabalho realizado por Dereby, que determina uma prevalência três vezes superior no diagnóstico de alergia em doentes com DM, comparativamente com a população geral. ${ }^{61}$

A enxaqueca ocorre com maior frequência em doentes com DM comparativamente com a população em geral. ${ }^{62,63}$ Uma associação entre a DM e a enxaqueca foi sugerida logo no primeiro relato de casos conhecidos de DM por Prosper Ménière. ${ }^{1}$ Desde então vários estudos realizados tentam relacionar a enxaqueca à DM. Wladislavosky-Waserman et al, reportam que 5\% dos casos identificados com DM apresentam enxaqueca. ${ }^{3}$ Hausler et al, encontram quatro crianças com ataques de enxaqueca clássica, num total de 14 crianças identificadas com DM. ${ }^{26}$ Radtke et al, mais recentemente, encontram um aumento na prevalência da enxaqueca em doentes com DM (56\%). ${ }^{62}$ Gopen et al determinam uma incidência da enxaqueca em doentes com DM de 4,5\%. ${ }^{63}$

\section{CONCLUSÃO}

Como se constata nos diversos estudos descritos anteriormente, tanto a prevalência como a incidência da DM apresentam uma grande variabilidade (prevalências entre 3,5 a 513 casos por 100000 habitantes; incidências entre 4,3 a 320 casos por 100000 habitantes), não se conseguindo estabelecer um valor real para cada uma desta variáveis. Por outro lado, podemos afirmar, com toda a certeza, que a prevalência e incidência da DM variam não só de país para país, mas também dentro de um mesmo país, e que os critérios mais ou menos restritos, a acessibilidade a instalações médicas, a população em estudo, o ano do estudo e os anos de follow-up em cada um dos estudos contribuem para um aumento ou diminuição dos valores obtidos para estas mesmas variáveis.

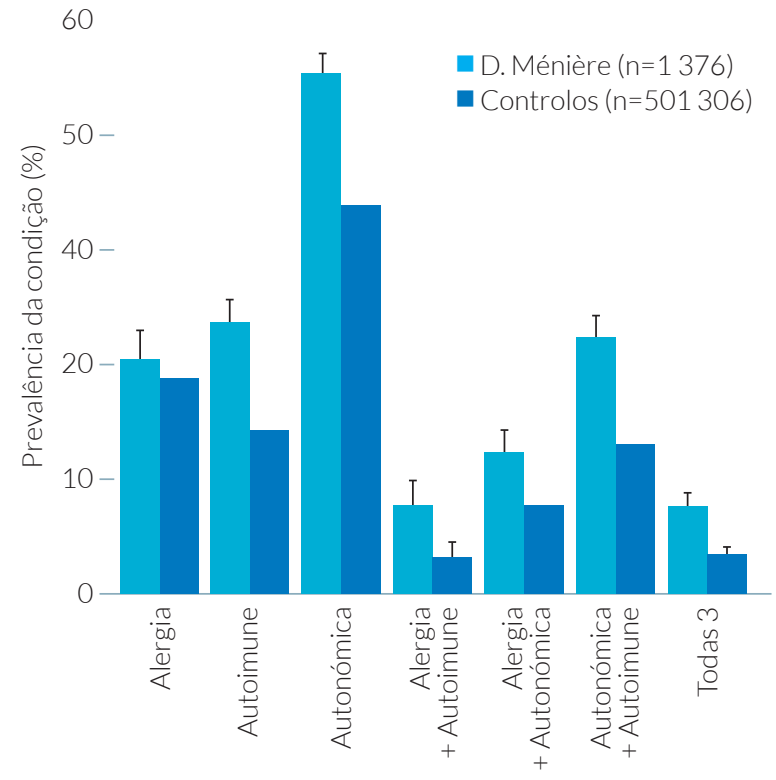

FIGURA 2. Prevalência, fatores associados e condições na doença de Ménière.

Adaptado de Tyrrell JS, et al. Prevalence, associated factors, and comorbid conditions for Ménière's disease. Ear Hear. 2014;35:e162-9.18

Na infância, a DM é extremamente rara. Contrariamente no idoso ela apresenta prevalências bastante altas, o que é expectável, sendo a DM uma doença crónica não letal. Quanto à existência de um maior predomínio da DM na raça caucasiana bem como um ligeiro aumento na prevalência no sexo feminino ela é aceite, de um modo geral, entre os vários investigadores.

A ocorrência da DM dá-se na meia-idade (quarta e quinta década). Sendo que o aparecimento da DM, na maioria dos casos, se manifesta apenas com um sintoma, existe um intervalo de tempo até ao diagnóstico definitivo ser realizado ( $\bar{\chi}=3$ anos). Nenhum dos ouvidos, direito ou esquerdo, parece apresentar uma maior predisposição para a DM.

Quanto à bilateralidade, existe uma tendência clara para a DM se tornar bilateral. Se nos estudos iniciais esta situação não se verificava, isso devia-se à janela de análise dos estudos publicados se basearem num curto espaço de tempo. Com o aparecimento mais recente de estudos em que existe um aumento dos anos de follow-up torna-se evidente um aumento de casos de DMB. Podemos mesmo afirmar que a percentagem de casos de DMB está correlacionada positivamente com o tempo de seguimento do estudo e inversamente com a rigidez aplicada nos critérios de diagnóstico selecionados.

Sendo o risco evidente de envolvimento do ouvido contralateral, mesmo em casos de longa duração de DMU, a terapêutica a aplicar deve sempre ser tida em conta tendo em mente um possível envolvimento do ouvido 
contralateral. O SI, a enxaqueca, a alergia e o SNA estão associados aos doentes com DM.

Tendo em conta os achados epidemiológicos anteriores da DM, parece patente a existência de uma predisposição genética. Vários estudos apontam que 3 a 20\% dos casos de DM é familiar. Em adição aos casos familiares, as diferenças na distribuição geográfica e diferentes incidências da DM, no género e raça, sugerem que os fatores genéticos são significativos. Uma frequência mais elevada de doenças autoimunes sistémicas observada neste grupo reforça, também ela, esta presunção.

Para finalizar, os doentes com DM, numa fase inicial, têm maior probabilidade de apresentarem uma má saúde mental, verificando-se uma redução no impacto dos sintomas em 85\% dos casos de DM à medida que os anos passam, nomeadamente através de mudanças no estilo de vida e tratamento médico ou cirúrgico. A informação relativa a uma melhoria no estado de saúde mental fornece uma mensagem positiva importante para os clínicos, bem como para os recém-diagnosticados casos de DM.

CONFLITOS DE INTERESSE: Os autores declaram a inexistência de conflitos de interesse na realização do trabalho.

FONTES DE FINANCIAMENTO: Não existiram fontes externas de financiamento para a realização deste artigo.

CONFLICTS OF INTEREST: The authors declare that they have no conflicts of interest.

FINANCIAL SUPPORT: This work has not received any contribution, grant or scholarship.

\section{REFERÊNCIAS}

1. Ménière P. Maladies de l'oreille interne offrant les symptômes de la congestion cérébrale apoplectiforme. Gaz Médicale Paris. 1861;16:88-9.

2. Sajjadi H, Paparella MM. Meniere's disease. Lancet. 2008;372:406-14.

3. Wladislavosky-Waserman P, Facer GW, Mokri B, Kurland LT. Meniere's disease: a 30-year epidemiologic and clinical study in Rochester, Mn, 1951-1980. Laryngoscope. 1984;94:1098-102.

4. Minor LB, Schessel DA, Carey JP. Meniere's disease. Curr Opin Neurol. 2004;17:9-16.

5. Harris JP, Alexander TH. Current-day prevalence of Ménière's syndrome. Audiol Neurotol. 2010;15:318-22.

6. Stahle J, Stahle C, Arenberg IK. Incidence of Ménière's disease. Arch Otolaryngol. 1978;104:99-102.

7. Arenberg IK, Balkany TJ, Goldman G, Pillsbury RC. The incidence and prevalence of Meniere's disease - a statistical analysis of limits. Otolaryngol Clin North Am. 1980;13:597-601.

8. American Academy of Ophtalmology and Otolaryngology, Committee on Hearing and Equilibrium, Subcommittee on Equilibrium and its Measurement. Meniere's disease: criteria for diagnosis and evaluation of therapy for reporting. Trans Am Acad Ophth Otolaryngol. 1972;76:1462-4.
9. American Academy of Otolaryngology - Head and Neck Surgery, Committee on Hearing and Equilibrium. Ménière's disease: criteria for diagnosis and evaluation of therapy for reporting. AAO-HNS Bull. 1985;5:6-7.

10. American Academy of Otolaryngology - Head and Neck Surgery, Committee on Hearing and Equilibrium. Committee on Hearing and Equilibrium guidelines for the diagnosis and evaluation of therapy in Menière's disease. American Academy of Otolaryngology-Head and Neck Foundation, Inc . Otolaryngol Head Neck Surg. 1995;113:181-5.

11. Watanabe Y, Mizukoshi K, Shojaku H, Watanabe I, Hinoki M, Kitahara M. Epidemiological and clinical characteristics of Menière's disease in Japan. Acta Otolaryngol Suppl. 1995;115:206-10.

12. Lopez-Escamez JA, Carey J, Chung WH, Goebel JA, Magnusson M, Mandala M, et al. [Diagnostic criteria for Meniere's disease. Consensus document of the Barany Society, the Japan Society for Equilibrium Research, the European Academy of Otology and Neurotology (EAONO), the American Academy of Otolaryngology-Head and Neck Surgery (AAO-HNS) and the Korean Balance Society]. Acta Otorrinolaringol Esp. 2016;67:1-7. Spanish.

13. Kotimäki J, Sorri M, Aantaa E, Nuutinen J. Prevalence of Meniere disease in Finland. Laryngoscope. 1999;109:748-53.

14. Cawthorne T, Hewlett AB. Ménière's Disease. Proc R Soc Med. 1954;47:25-38.

15. Havia M, Kentala E, Pyykkö I. Prevalence of Menière's disease in general population of Southern Finland. Otolaryngol Head Neck Surg. 2005;133:762-8.

16. Suárez AR, Angulo CM. Concepto e epidemiología de la enfermedad de Ménière. In: Garriguez HP, Escámez JA, Angulo CM, Fernández NP, editors. Enfermedad de Ménière: desde las ciencias básicas hacia la medicina clínica. Badalona (Spain): Schering-P; 2009. p. 115-22.

17. Radtke A, von Brevern M, Feldmann M, Lezius F, Ziese T, Lempert T, et al. Screening for Menière's disease in the general population - the needle in the haystack. Acta Otolaryngol. 2008;128:272-6

18. Tyrrell JS, Whinney DJD, Ukoumunne OC, Fleming LE, Osborne NJ. Prevalence, associated factors, and comorbid conditions for Meniere's disease. Ear Hear. 2014;35:e162-9.

19. Naito T. Clinical studies on Ménière's disease. Rev Laryngol Otol Rhinol. 1962;83:361-83.

20. Nakae K, Nitta H, Hattori Y, Maeda K, Watanabe I, Mizukoshi $K$, et al. The prevalence of Ménière's disease in Japan. Pract Otorhinolaryngol. 1980;73:1023-9.

21. Shojaku H, Watanabe Y, Fujisaka M, Tsubota M, Kobayashi K, Yasumura S, et al. Epidemiologic characteristics of definite Ménière's disease in Japan. A long-term survey of Toyama and Niigata prefectures. ORL J Otorhinolaryngol Relat Spec. 2005;67:305-9.

22. Celestino D, Ralli G. Incidence of Menière's disease in Italy. Am J Otol. 1991;12:135-8.

23. Okafor BC. Incidence of Menière's disease. J Laryngol Otol. 1984;98:775-9.

24. Brobby GW. The incidence of Meniere's disease in Kumasi-Ghana. Ghana Med J. 1992;26:454-9.

25. Ibekwe TS, Ijaduola GTA. Meniere's disease: rare or underdiagnosed among Africans. Eur Arch Otorhinolaryngol. 2007;264:1399-403.

26. Hausler R, Toupet M, Guidetti G, Basseres F, Montandon P. Ménière's Disease in Children. Am J Otolaryngol. 1987;8:187-93.

27. Meyerhoff WL, Paparella MM, Shea D. Ménière's disease in children. Laryngoscope. 1978;88:1504-11. 
28. Sadé J, Yaniv E. Meniere's disease in infants. Acta Otolaryngol. 1984;97:33-7.

29. Brantberg K, Duan M, Falahat B. Ménière's disease in children aged 4-7 years. Acta Otolaryngol. 2012;132:505-9.

30. Akagi H, Yuen K, Maeda Y, Fukushima K, Kariya S, Orita Y, et al. Ménière's disease in childhood. Int J Pediatr Otorhinolaryngol. 2001;61:259-64.

31. Ballester M, Liard P, Vibert D, Häusler R. Menière's disease in the elderly. Otol Neurotol. 2002;23:73-8.

32. Friberg U, Stahle J, Svedberg A. The natural course of Meniere's disease. Acta Otolaryngol Suppl. 1984;406:72-7.

33. Balkany TJ, Sires B, Arenberg IK. Bilateral aspects of Meniere's disease: an underestimated clinical entity. Otolaryngol Clin North Am. 1980;13:603-9

34. Morales Angulo C, Gómez Castellanos R, García Mantilla J, Bezos Capelastegui JT, Carrera F. Epidemiologia de la enfermedad de Meniere en Cantabria. Acta Otorrinolaringol Esp. 2003;54:601-5. Spanish.

35. House JW, Doherty JK, Fisher LM, Derebery MJ, Berliner KI. Meniere's disease: prevalence of contralateral ear involvement. Otol Neurotol. 2006;27:355-61.

36. Paparella MM, Griebie MS. Bilaterality of Meniere's disease. Acta Otolaryngol. 1984;97:233-7.

37. Enander A, Stahle J. Hearing in Menière's disease. A study of pure-tone audiograms in 334 patients. Acta Otolaryngol. 1967;64:543-56.

38. Thomas K, Harrison MS. Long-term follow up of 610 cases of Ménière's disease. Proc R Soc Med. 1971;64:853-7.

39. Stahle J, Bergman B. The caloric reaction in Meniere's disease. An electronystagmographical study in 300 patients. Laryngoscope. 1967;77:1629-43.

40. Stahle J. Advanced Meniere's disease. A study of 356 severely disabled patients. Acta Otolaryngol. 1976;81:113-9.

41. Ohmen JD, White CH, Li X, Wang J, Fisher LM, Zhang H, et al. Genetic evidence for an ethnic diversity in the susceptibility to Meniere's disease. Otol Neurotol. 2013;34:1336-41.

42. Caparosa RJ. Medical treatment for Meniere's disease. Laryngoscope. 1963;73:666-72.

43. Kitahara M, Futaki T, Nakano K. Ethnic Aspect of Meniere's Disease. Equilib Res Suppl. 1971;1:104-10.

44. Morrison AW, Bailey MES, Morrison GAJ. Familial Ménière's disease: clinical and genetic aspects. J Laryngol Otol. 2009;123:29-37.

45. Birgerson L, Gustavson KH, Stahle J. Familial Menière's disease: a genetic investigation. Am J Otol. 1987;8:323-6.

46. Morrison AW, Johnson KJ. Genetics (molecular biology) and Meniere's disease. Otolaryngol Clin North Am. 2002;35:497516.

47. Gazquez I, Soto-Varela A, Aran I, Santos S, Batuecas A, Trinidad $\mathrm{G}$, et al. High prevalence of systemic autoimmune diseases in patients with meniere's disease. PLoS One. 2011;6:e26759.
48. Mizukoshi K, Ino H, Ishikawa K, Watanabe Y, Yamazaki H, Kato I, et al. Epidemiological survey of definite cases of Meniere's disease collected by the seventeen members of the Meniere's Disease Research Committee of Japan in 1975--1976. Adv Otorhinolaryngol. 1979;25:106-11.

49. Klockars T, Kentala E. Inheritance of Meniere's disease in the Finnish population. Arch Otolaryngol Head Neck Surg. 2007;133:73-7.

50. Morrison AW. Anticipation in Menière's disease. J Laryngol Otol. 1995;109:499-502.

51. Oliveira CA, Ferrari I, Messias Cl. Occurrence of familial Meniere's syndrome and migraine in Brasília. Ann Otol Rhinol Laryngol. 2002;111:229-36.

52. Fung K, Xie Y, Hall SF, Lillicrap DP, Taylor SAM. Genetic basis of familial Meniere's disease. J Otolaryngol. 2002;31:1-4.

53. Lee JM, Kim MJ, Jung J, Kim HJ, Seo YJ, Kim SH. Genetic aspects and clinical characteristics of familial meniere's disease in a South Korean population. Laryngoscope. 2015;15:217580.

54. Coker NJ, Coker RR, Jenkins HA, Vincent KR. Psychological profile of patients with Menière's disease. Arch Otolaryngol Head Neck Surg. 1989;115:1355-7.

55. Friberg U, Stahle J. The epidemiology of Ménière's disease. In: Harris JP, editor. Meniere's Disease. The Hague: Kugler Publications; 1999. p. 17-28.

56. Watanabe I. Incidence of Ménière's Disease, including some other epidemiological data. In: Oosterveld WJ, editor. Meniere's Disease: A Comprehensive Appraisal. New York: Wiley-Blackwell; 1983. p. 9-23.

57. Derebery MJ, Berliner KI. Allergy and its relation to Meniere's disease. Otolaryngol Clin North Am. 2010;43:1047-58.

58. Brenner M, Hoistad DL, Hain TC. Prevalence of thyroid dysfunction in patients with Ménière's disease. Arch Otolaryngol Head Neck Surg. 2004;130:226-8.

59. Duke WW. Ménière's Syndrome caused by Allergy. JAMA. 1923;81:2179-81.

60. Derebery MJ. Allergic and immunologic aspects of Meniere's disease. Otolaryngol Head Neck Surg. 1996;114:360-5.

61. Derebery MJ. Allergic management of Meniere's disease: an outcome study. Otolaryngol Head Neck Surg. 2000;122:17482.

62. Radtke A, Lempert T, Gresty MA, Brookes GB, Bronstein AM, Neuhauser $\mathrm{H}$. Migraine and Ménière's disease: is there a link? Neurology. 2002;59:1700-4.

63. Gopen Q, Viirre E, Anderson J. Epidemiologic study to explore links between Ménière syndrome and migraine headache. Ear Nose Throat J. 2009;88:1200-4. 